\title{
miR-15a Targets ABAT Gene to Regulate the Differentiation of Preadipocytes of Yanbian Yellow Cattle
}

\section{Jinhui Bai}

Yanbian University Agriculture College

Hongyan Xu

Yanbian University Agriculture College

Jiachen Fang

Hirosaki University: Hirosaki Daigaku

Congcong Zhang

Yanbian University Agriculture College

Jixuan Song

Yanbian University Agriculture College

Xinxin Zhang

Yanbian University Agriculture College

\section{Beibei Hao}

Yanbian University Agriculture College

\section{Baozhen Yin}

Yanbian University Agriculture College

GuangjunXia ( $\nabla$ ybuac@ybu.edu.cn)

Yanbian University Agriculture College

\section{Research}

Keywords: Yanbian Yellow Cattle, Preadipocytes, miR-15a, ABAT gene

Posted Date: November 23rd, 2021

DOI: https://doi.org/10.21203/rs.3.rs-1092736/v1

License: (c) (1) This work is licensed under a Creative Commons Attribution 4.0 International License.

Read Full License 


\section{Abstract}

\section{Background}

MicroRNAs (miRNAs) are small RNAs that cannot be translated into proteins. The length of a mature miRNA is approximately 20-25 nt. Neural regulation, inflammatory response, cell proliferation and apoptosis, fat metabolism, and cell differentiation, among others, have become research hotspots in recent years. In this experiment, dual-luciferase reporter gene detection, qRT-PCR, oil red 0 staining, western blotting, and other methods were used to select the differentially expressed miR-15a and its target gene, the 4-aminobutyrate aminotransferase (ABAT) gene, in the longissimus muscle tissues of cattle bulls and steers. By transfecting Yanbian yellow cattle precursor adipocytes with equal amounts of artificially synthesised miR-15a mimics, mimics NC, miR-15a inhibitor, and inhibitor NC and inducing differentiation, the total RNA and total protein of cells during the induced differentiation process were extracted.The effect of miR-15a on the mRNA and protein expression of adipogenesis-related genes was analysed.

\section{Results}

The results showed that the lipid droplet content and lipid droplet size of the miR-15a mimic group were significantly lower than those of the mimics NC group, and the mRNA levels of the peroxisome proliferator-activated receptor $\mathrm{Y}$ (PPARY) and CCAAT enhancer-binding protein a (C/EBPa) genes in the miR-15a mimics group were significantly lower than those in the mimics NC group. At the same time, the protein expression of miR-15a mimic group cells was also significantly lower than that of the NC group cells. Meanwhile, the mRNA levels of the PPARY and C/EBPa genes of miR-15a inhibitor group cells were significantly higher than those of the NC group cells, and the protein expression levels were also significantly higher than those of the NC group cells. The luciferase reporter results showed that miR-15a mimics significantly inhibited the activity of the ABAT-3'UTR dual luciferase reporter gene vector, indicating that the miR-15a and ABAT genes have a targeting effect, and that overexpressed miR-15a significantly reduced the expression of the ABAT gene in the cell, inhibited the expression of miR-15a, and significantly increased the expression of the ABAT.

\section{Conclusions}

In summary, miR-15a may target the ABAT gene to regulate the differentiation of Yanbian yellow cattle precursor adipocytes.

\section{Background}

Beef is loved by people because of its rich nutrition and unique taste. The quality of beef is related to intramuscular fat content. Intramuscular fat content can often affect a person's choice of meat products [1]. Yanbian Yellow Cattle is one of China's five major local fine breeds of cattle. This breed of cattle has 
the characteristics of cold tolerance, rough feeding, strong disease resistance, and tender and delicious meat.

MicroRNAs (miRNAs), that were first discovered by R. Lee in Caenorhabditis elegans in 1993, are widely expressed in eukaryotic cells [2]. miRNAs are transcribed.from the genomic DNA, but not translated into proteins(i.e., they are noncoding). Instead, they bind to the target mRNAs and regulate expression of target genes by inhibiting translation of the transcribed mRNAs or causing their degradation [3]. Studies have found that miRNAs are involved in the expression of approximately $60 \%$ of genes in mammals and $30 \%$ of human genes. miRNAs are involved in many biological metabolic processes, including cell growth, apoptosis, signal transduction, insulin resistance, and adipocyte differentiation [4]. In recent years, studies have found that miRNAs not only play a key role in fat deposition and metabolism in cattle, but also play a significant role in the production, proliferation, and differentiation of adipocytes [5]. Fan et al. [6] screened and determined that miR-193a-5p regulates the metabolism of unsaturated fatty acids in bovine mammary epithelial cells by targeting the FADS1 gene, and that the expression of triglycerides was significantly positively correlated with the increase or decrease of FADS1. In contrast to miR-193a-5p, Jin et al. [7] reported that the expression of 18 miRNAs was related to the thickness of the back fat of the steers, and that the expression of miR-378 was significant. Wang et al. [8] found that miR-27a-3p targets PPARy to inhibit triglyceride synthesis in cow mammary epithelial cells. Chen et al. [9] demonstrated that bta-miR-376a can target Kruppel-like transcription factor 15 (KLF15) to negatively regulate the proliferation and proliferation of Qinchuan bovine adipocytes through luciferase activity experiments, oil red $O$ staining, and triglyceride content analysis. Differentiation is a new regulator of the development of Qinchuan beef cattle.

MiR-15a is an miRNA closely related to the functions of blood vessels and endothelial cells. At present, most studies on miR-15a focus on human diseases, and there are few studies on animal fat regulation. MiR-15a plays a significant role in the production of insulin by pancreatic $\beta$-cells [10]. Overexpression of miR-15a in pancreatic $\beta$-cells cultured in vitro under high-glucose conditions can activate oxidative stress targeting AKT3 and cause cell apoptosis. Overexpression of miR-15a can reduce the expression of E2F3 gene, inhibit the proliferation of chicken macrophages, and cause apoptosis and G1/S phase arrest [11]. The knockout of endogenous miR-15a after insulin treatment can inhibit glucose transporter 4 (GLUT4) translocation to the cell membrane to increase extracellular glucose. However, the overexpression of miR$15 a$ has no significant effect on glucose metabolism [12].

The ABAT gene, 4-aminobutyrate aminotransferase, encodes the ABAT protein $\gamma$-aminobutyrate aminotransferase, which has an impact on the function of mitochondrial membranes and the activity of mitochondrial respiratory chain complexes. Mammalian nerve transmitter metabolism, the synthesis of various substances, cell proliferation, differentiation, and other functions are all involved and regulated by the ABAT gene $[13,14]$. Sun et al. [15] conducted an integrated microRNA-mRNA analysis of the muscle tissue of dried Chinese meat Merino sheep and small tail Han sheep which revealed that the ABAT gene can promote the processes of insulin secretion and carbohydrate and amino acid metabolism in sheep. In vitro cell culture experiments found that the expression of ABAT gene in the eye muscles of dried Chinese 
meat Merino sheep was higher than that in small-tailed Han sheep. The results also showed that oar-miR381-5p targeted regulation of ABAT gene expression affects the growth and development of sheep muscle, and has a certain regulatory effect on meat quality traits. Xia Guangjun [16] also found that the ABAT gene may be the target gene of the differentially expressed miRNAs in Yanbian yellow cattle and steers, indicating that the ABAT gene may be involved in the regulation of Yanbian yellow beef quality traits.

With the advancement of science and technology, the development of society, and the improvement of the living standards of residents, an increasing number of people are paying more attention to the quality and safety of meat. To improve the quality of beef, increase the production of high-end beef, and accelerate the cultivation of specialised beef cattle breeds, researchers have used a variety of advanced technologies to improve the quality of beef and have achieved satisfactory results. In this study, we used in vitro cell culture and molecular biology techniques to investigate the effects of miR-15a on Yanbian cattle precursor adipocytes by transfecting miR-15a mimics, mimics NC, miR-15a inhibitor, and inhibitor $\mathrm{NC}$ into Yanbian cattle precursor adipocytes. The role and regulation mechanism of differentiation provide a theoretical basis and supporting data for the study of Yanbian yellow beef at the molecular and cellular levels.

\section{Methods}

\section{Cell culture and adipogenic differentiation}

Yanbian yellow cattle preadipocytes were obtained from the Jilin Gongzhuling Animal Husbandry Branch, while human hepatocyte HepG2 cells were obtained from the Yanbian University School of Pharmacy. The experimental procedures were approved by the Yanbian University Animal Care and Use Committee (Approval ID: 201702). The cells were cultured at $37^{\circ} \mathrm{C}$ in a $5 \% \mathrm{CO}^{2}$ incubator in DMEM (Gibco, China) supplemented with $10 \%$ foetal bovine serum (FBS) (BI, Israel). When the preadipocytes reached $80 \%$ confluence, they were placed into 6 -well plates. When the cell confluence reached $100 \%$, the culture medium was replaced with DMEM containing $1 \mathrm{mg} / \mathrm{L}$ insulin, $1 \mu \mathrm{mol} / \mathrm{L}$ 3-isobutyl-1-methylxanthine, 1000 $\mu \mathrm{mol} / \mathrm{L}$ dexamethasone (Sigma-Aldrich, China), and 10\% FBS. After two days, the medium was replaced with DMEM containing $10 \mu \mathrm{g} / \mathrm{mL}$ insulin and 10\% FBS, and then replaced with DMEM containing $10 \%$ FBS after two days. The cells were cultured for nine days.

\section{Bioinformatics}

The sequence of mature bta-miR-15a was obtained from miRBase (http://www.mirbase.org/), and the target genes were predicted using TargetScan (http://www.mirdb.org/).

\section{Transfection of bovine preadipocytes}

Bovine preadipocytes were seeded into 6-well plates at a density of $1 \times 10^{6}$ cells/well in $2 \mathrm{~mL}$ of DMEM containing 10\% FBS. For transfection, $5 \mu \mathrm{L}$ of LipofectamineTM 2000 (11668019, Invitrogen, China) and 
$10 \mu \mathrm{L}$ of $20 \mu \mathrm{M}$ of bta-miR-15a mimics or an equivalent amount of negative control (NC) mimics, miRNA inhibitor, or NC inhibitor were added to $300 \mu \mathrm{L}$ of Opti-MEM medium (Gibco, USA) added to the cells in DMEM-containing 10\% FBS. All miRNAs were purchased from Jiangsu GenePharma (Suzhou, China). The miRNA sequences are listed in Table S1 in the Supplementary Materials.

\section{Dual-luciferase reporter assay}

Based on the 3'UTR sequence of the bovine ABAT gene provided by the National Center for Biotechnology Information (NCBI), we designed primers for the amplification of a gene fragment that perfectly matched the bta-miR-15a seed sequence (2nd to 8 th nucleotides at the 5 -end of the miRNA). The sequences coding for the Xhol and Pmel restriction endonuclease sites were added to the 5 -ends of the forward and reverse primers, respectively. The obtained PCR product was ligated into the pmirGLO vector (Promega, China). Mutations to the putative binding site ( 3 nucleotides) were introduced using mutation primers; the mutant sequences were amplified by PCR and cloned into the pmirGLO vector. The primer sequences are listed in Table S2 in the Supplementary Materials.

HepG2 cells were seeded into 96-well plates and cotransfected with $0.1 \mu \mathrm{g}$ of dual-luciferase reporter vector $(0.5 \mu \mathrm{g})$ and $20 \mu \mathrm{M}$ bta-miR-15a or NC mimics using Lipofectamine ${ }^{\mathrm{TM}} 3000$ (Invitrogen). At $48 \mathrm{~h}$ post-transfection, luciferase activity was detected using the Dual-Glo ${ }^{T M}$ Luciferase Assay System (Promega) and quantified using a luminometer (GloMax 20/20, Promega).

\section{Oil Red 0 staining and triglyceride assay}

The cells were washed twice with PBS and fixed in situ in a $10 \%$ paraformaldehyde solution at $22 \pm 3^{\circ} \mathrm{C}$ for $30 \mathrm{~min}$. The fixed cells were rinsed twice with phosphate-buffered saline (PBS). Oil Red 0 (Sigma-Aldrich) stock solution ( $0.5 \mathrm{~g}$ of Oil Red 0 dissolved in $100 \mathrm{~mL}$ of isopropanol and filtered) was mixed with sterile water at a $3: 1$ ratio and added to the cells in a 6-well plate for staining at $22 \pm 3^{\circ} \mathrm{C}$ for $40 \mathrm{~min}-1 \mathrm{~h}$. Next, the Oil Red $\mathrm{O}$ solution was discarded, and the cells were washed four times with water and observed under a microscope. The triglyceride assay kit (Nanjing Jiancheng Bioengineering Institute, Nanjing, China) was used to quantify the triglyceride content in adipocytes.

\section{RNA extraction and RT-qPCR analysis}

Total RNA and miRNAs were extracted using the TRIzol Universal and miRcute miRNA isolation kit, respectively (Tiangen, China). RNA was reverse transcribed into CDNA using the PrimeScript RT reagent kit with genomic DNA Eraser (Takara, China). RT-qPCR was performed using the TB GreenTM Premix Ex Taq II kit (Takara). For miRNA quantification, reverse transcription and qPCR were performed using the miRcute Plus miRNA First-Strand cDNA Kit and miRcute Plus miRNA qPCR Kit (SYBR Green), respectively (Tiangen) (Tiangen). RT-qPCR reactions were performed using a PCRmax Eco 48 real-time PCR machine (PCRmax, UK). The relative mRNA and miRNA expression levels were calculated using this method. The actin gene was used as a housekeeping gene to normalise the expression of protein-coding genes. The sequences of the primers used for qPCR are shown in Table S3 in the Supplementary Materials.

\section{Protein extraction and Western blotting}


The cells were lysed by incubation with RIPA lysis buffer (Beyotime, China) containing 1 mM PMSF (Beyotime) on ice. The total protein concentration in the cell lysates was measured using the Enhanced BCA Protein Assay Kit (Beyotime) according to the manufacturer's instructions. Protein samples were subjected to electrophoresis in $12 \%$ SDS-polyacrylamide gel ( $20 \mu \mathrm{g}$ protein per lane) and transferred to polyvinylidene difluoride (PVDF) membranes (Millipore, USA) according to the manufacturer's instructions (Bio-Rad, USA). The PVDF membrane was rinsed with TBS containing $0.1 \%$ Tween 20 (TBST) and blocked with $5 \%$ ovalbumin (Solarbio, China) in TBST for $2 \mathrm{~h}$ at $22 \pm 3^{\circ} \mathrm{C}$. The membrane was then incubated with the primary antibodies in blocking buffer overnight at $4^{\circ} \mathrm{C}$, washed five times with TBST, and incubated with horseradish peroxidase-conjugated secondary antibodies at $4^{\circ} \mathrm{C}$ for $2 \mathrm{~h}$. After rinsing five times with TBST, the immunoblots were developed using a chemiluminescence solution and analysed with an Alliance MINI HD9 AUTO Western Blot Imaging System (UVITEC, USA). The intensities of the target protein bands were normalised to the intensities of the -actin bands and computed using the ImageJ program.

\section{Statistical analysis}

All results are presented as the mean \pm SD. The data were evaluated with the Student's t-test (RT-qPCR, triglyceride assay) or one-way analysis of variance (one-way ANOVA) (dual-luciferase reporter assay). Differences between groups were considered statistically significant at $P<0.05$. All statistical analysis procedures were performed using SPSS 20.0.

\section{Results}

\section{miR-15a is highly conserved in mammals.}

bta-miR-15a is encoded on chromosome 7 and produces only one mature miRNA. Interestingly, bta-miR15a, ccs-miR-15a, and cfa-miR-15a are homologous. These mature miRNAs' sequences are also highly conserved among mammals, including gorillas, monkeys, pigs, and goats. Although there are slight differences in the mature miR-15a sequence between mammals, the seed sequence was the same (Fig. 1).

\section{miR-15a can regulate the differentiation of Yanbian yellow cattle precursor adipocytes.}

The Yanbian yellow cattle precursor adipocytes were transfected with miRNA containing FAM green fluorescent labelling, and the transfection efficiency of miR-15a was observed under a microscope $4 \mathrm{~h}$ later. MiR-15a has higher transfection efficiency(Fig. 2a). Next, differentiation was induced. After differentiation, the miR-15a mimic group inhibited lipid droplet production in preadipocytes compared to the NC control group (Fig. 2b). The cells on the 9th day of induced differentiation were collected, and the TG content in the cells was determined using a TG kit. It can be seen from the figure $2 \mathrm{c}$ that the triglyceride content of the miR-15a mimic group was significantly lower than that of the mimics NC group $(P<0.001)$. Furthermore, the inhibition rate reached $76.6 \%$, indicating that the overexpression of miR-15a can inhibit the differentiation of bovine preadipocytes and reduce the amount of TG released by the cell 
(Fig. 2c). Total RNA was extracted from cells on the 0th, 2nd, 4th, 6th, and 8th days of the adipogenic differentiation stage of Yanbian yellow cattle and analysed the expression of adipogenic marker genes. The expression of PPARy and C/EBPa, which was the highest on the 4th day (Fig. 2d), induced differentiation. Therefore, on the fourth day of differentiation of precursor adipocytes, the total RNA and total protein of cells transfected with the miR-15a mimic and mimics NC groups were extracted, and the lipid metabolism marker genes PPARy, PPARy, and PPARy were detected by fluorescent quantitative PCR and western blotting. The relative expression of C/EBPa at the mRNA and protein levels was measured. The mRNA and protein expression levels of PPARY and C/EBPa genes in the cells transfected with btamiR-15a mimics were significantly lower than those in the mimics NC group $(P<0.05)$ (Fig. 2e,g). In contrast, the mRNA and protein expression of PPARY and C/EBPa genes in the miR-15a inhibitor group were significantly higher than those in the inhibitor NC group $(P<0.05)(F i g .2 f, h)$.

\section{miR-15a targets the ABAT gene of cattle.}

In this experiment, a fragment containing the miR-15a seed sequence binding site in the 3'UTR region of ABAT was amplified, and the length of the product was $500 \mathrm{bp}$. Using NCBI's BLAST to compare the ABAT wild-type cloned sequence with the cattle's ABAT gene (NM_001081581), it was found that the base pairing rate reached $100 \%$, and the wild-type vector (Psicheck2-ABAT-miR-15a) was successfully constructed (Fig. 3a). The mutant vector "Psicheck2-MUT" ((Fig. 3b) A-B) was constructed based on the mRNA binding site of miR-15a and the bovine ABAT gene and divided into six groups: miR-15a mimics, mimics NC control, wild-type, mutant, and empty plasmids. These groups were co-transfected into HepG2 cells, and the fluorescence activity was measured for $24 \mathrm{~h}$ (Fig. 3c). The fluorescence activity of the wildtype and miR-15a mimics group was significantly lower than that of the mimics NC group $(P<0.05)$, indicating that miR-15a and ABAT wild-type vector had a binding effect, while the mimics NC group and the wild-type and mutant vectors had a binding effect. There was no significant change in fluorescence activity $(P>0.05)$. This result indicates that miR-15a targets the 3 'UTR of the ABAT gene in cattle and has a regulatory effect on the gene.

\section{miR-15a targets ABAT gene to regulate the differentiation of Yanbian yellow cattle adipocytes.}

The total RNA of Yanbian yellow cattle precursor adipocytes was extracted on the 0th, 2nd, 4th, 6th, and 8th days of differentiation, and the expression of miR-15a target gene ABAT was detected by qRT-PCR during different differentiation induction time periods. The results showed that the expression level of the ABAT gene was highest on the 6th day of adipogenic differentiation (Fig. 4a). To determine whether bovine miR-15a regulates adipocyte differentiation by targeting the expression of the ABAT gene, we extracted the total RNA and total cell protein of Yanbian yellow cattle precursor adipocytes on the 6th day of induced differentiation for detection, and the mRNA expression of the ABAT gene. The amount and protein expression in the preadipocytes of Yanbian yellow cattle in the miR-15a mimics group were significantly lower than those in the mimics NC group $(P<0.05)$ (Fig. $4 b, c)$. To verify the regulatory effect of miR-15a on the ABAT gene, miR-15a inhibitor and inhibitor NC were transfected into pre-adipocytes for detection. The results showed that the mRNA and protein expression of the ABAT gene in the Yanbian 
yellow cattle precursor adipocytes of the miR-15a inhibitor group were significantly higher than those of the inhibitor NC group $(P<0.05)$ (Fig. 4d, e). This further confirms that miR-15a targets the ABAT gene to regulate the differentiation of Yanbian yellow cattle adipocytes, and miR-15a negatively regulates the expression of the ABAT gene.

\section{miR-15a participates in AMPK-mTOR-Wnt pathway to regulate the differentiation of Yanbian yellow cattle precursor adipocytes.}

In this experiment, the same number of bta-miR-15a mimics, mimics NC, bta-miR-15a inhibitor, and inhibitor NC were transfected to detect the mRNA and protein levels of FAS, SREBP1, ACC, ADPN, mTOR, and AMPKa in cells. The results showed that overexpression of miR-15a significantly inhibited the expression of mTOR, thereby reducing the expression of its downstream gene SREBP1. This causes the expression of FAS and ACC genes and differentiation of preadipocytes to be inhibited. In addition, overexpression of miR-15a significantly reduced the mRNA and protein expression of ADPN and AMPKa genes. On the other hand, inhibiting the expression of miR-15a significantly increases the expression of FAS, SREBP1, ACC, ADPN, and mTOR genes in the mRNA and protein in the cell. Furthermore, inhibiting the expression of miR-15a also increases the mRNA expression of AMPKa $(P>0.05)$, and significantly increases the protein expression of AMPKa $(P<0.05)$. The reason for this result may be the degradation of RNA. The results showed that miR-15a downregulated the expression of ADPN and mTOR genes, reduced the expression of SREBP1 and AMPKa, and inhibited the expression of ACC and FAS genes, thereby inhibiting the differentiation of Yanbian yellow cattle precursor adipocytes (Fig. 5a,b).

\section{Discussion}

Yanbian Yellow Cattle occupies a superior position in market competition because of its unique meat flavour and good meat production performance. Currently, it is a well-known beef cattle breed in China. Cell culture in vitro has become a popular research method because it can understand the complete life activities of cells at the molecular level [17]. Through the cultivation and induction of differentiation of precursor adipocytes, we can more clearly understand the occurrence of adipose tissue, while through molecular biology technology, we can understand the effect of various regulatory factors on the entire process of adipogenesis. In recent years, animal husbandry and obesity have attracted much attention in recent years [18]. miRNAs are important regulators of fat formation and lipid metabolism. Xu et al. [19] cultured Yanbian yellow cattle precursor adipocytes in vitro and transfected miRNA and found that miR1271 could inhibit the expression of the ATF3 gene and promote the adipogenic differentiation of precursor adipocytes. In addition, miR-381 can also inhibit the expression of the KCTD15 gene [20]. Wang et al. [21] found that the overexpression of miR-223 in chicken liver cells LMH reduced the mRNA and protein expression of DAGLA. Meanwhile, knocking out miR-223 increased the expression of DAGLA in LMH cells, indicating that miR-223 targets DAGLA and downregulates its expression. Lee et al. [22] found that miR-15a was upregulated in the longissimus dorsi muscle of Korean cattle. Li et al. [23] found that miR-15a-3p and miR-15b-3p have significant differences in expression between healthy cows and mastitis-infected cows in the analysis of miRNAs related to the regulation of the immune response of 
cattle. Li et al. [24] found that bta-miR-15a inhibited the expression of casein in bovine mammary epithelial cells and reduced the number and viability of cells. In addition, it inhibited the activity of mammary epithelial cells and the expression of mRNA and protein of GHR, suggesting that bta- miR-15a may play a significant role in the physiological process of mammary glands. This experiment was based on the differential expression of miR-15a in the longissimus dorsi muscle tissue of Yanbian cattle and steers screened by a previous research group. We transfected the same amount of bta-miR-15a into the Yanbian cattle precursor adipocytes. After inducing differentiation using the mimics NC, bta-miR-15a inhibitor, and inhibitor NC, the total RNA and total protein of the cells were extracted, and the changes in the expression of PPARY and C/EBPa mRNA and the changes in protein expression were detected. The results showed that the mRNA and protein expression of lipid metabolism marker genes PPARY and $\mathrm{C} / \mathrm{EBPa}$ in adipocytes in the miR-15a mimics group were significantly lower than those in the mimics NC group, and the results were reversed after inhibiting their expression. After differentiation, the degree of lipid accumulation in the cells was detected by Oil Red $O$ staining, and the TG content was measured using a microplate reader. It was confirmed that miR-15a reduced the number of stained mature adipocytes and the secretion of TG. Wu Xulun [25] studies have shown that inhibiting $\beta$-catenin protein phosphorylation may inhibit the expression of PPARY and C/EBPa and then participate in the Wnt/ $\beta$ catenin signalling pathway to inhibit adipogenic differentiation and lipid droplet generation, which is also consistent with the results of this experiment. These results indicate that miR-15a may regulate the differentiation of Yanbian yellow cattle precursor adipocytes.

The ABAT gene, encoded by the ABAT protein, is $y$-aminobutyric acid aminotransferase, which can maintain the function of the mitochondrial membrane and the activity of mitochondrial respiratory chain complex. It is important in mammalian neurotransmitter metabolism, substance synthesis, and cell function regulation $[13,14]$. Sun et al. [15] revealed that the ABAT gene is involved in biological processes, such as the positive regulation of insulin secretion, carbohydrate metabolism pathways, amino acids, and metabolic pathways. Moreover, through in vitro cell culture, it was found that the ABAT gene was highly expressed in the longissimus dorsi of dried Chinese meat Merino sheep, and lowly expressed in the longissimus dorsi of small-tailed Han sheep. It is inferred that in sheep muscle tissue, oar- miR-381-5p regulates the ABAT gene by targeting to play a regulatory role in sheep muscle growth and meat quality. Therefore, the mechanism by which ABAT regulates lipid metabolism and adipogenic differentiation requires further study and exploration. Therefore, we quantified the expression of ABAT during the differentiation of Yanbian yellow cattle preadipocytes. It was found that the ABAT mRNA level reached its lowest level on the 6th day from the initial stage of preadipocyte differentiation, and then gradually increased until adipocytes were formed. This result indicates that ABAT may play a significant role in the early differentiation of Yanbian yellow cattle preadipocytes. In addition, we used dual-luciferase reporter gene detection to prove that miR-15a overexpression significantly inhibited the activity of the ABAT-3'UTR dual-luciferase reporter gene vector, indicating that miR-15a can have a targeting effect with the ABAT gene. At the same time, overexpression of miR-15a significantly reduced the expression of the ABAT gene in the cell, while inhibiting the expression of miR-15a significantly increased the expression of the ABAT gene will increase significantly. 
The differentiation of preadipocytes into adipocytes is a complex process, accompanied by various transcription factors, including C/EBPs, PPARs, SREBP family, and cytokines related to fat synthesis, including ACC and FABP4, to strictly regulate the differentiation process. Studies have shown that mTOR, Wnt/ $\beta$-catenin, AMPK, and other signalling pathways are involved in fat synthesis and metabolism [26]. SREBP-1c is a key gene in the mTOR pathway and is an essential element of lipid and cholesterol metabolism [27]. C/EBPa can promote its expression, and SREBP-1c can bind to the promoter of PPARY to activate the expression of PPARY [28]; adipokine secretion is also related to the TG content of adipocytes. Lipid accumulation leads to decreased ADPN secretion [29]. AMPK can stimulate the activation of ADPN and other cytokines to regulate cell lipid metabolism. AMPK can also inhibit TG synthesis and stimulate fatty acid oxidation and mitochondrial biosynthesis, inhibit the expression of ACC and reduce its activity, and reduce the accumulation of intracellular lipids [30]. Yao et al. [31] found that interfering with the expression of miR-15a in macrophages can reduce the phosphorylation levels of AKT and mTOR, indicating that it can participate in the PI3K/Akt/mTOR signalling pathway to inhibit the migration and invasion of glioma cells. Zhang et al. [32] found that miR-15a-5p induces pulmonary artery smooth muscle cell apoptosis in animal models of pulmonary hypertension through the VEGF/p38/MMP2 signalling pathway. In this experiment, to explore the regulatory mechanism of miR-15a on the differentiation of Yanbian yellow cattle adipocytes, the same number of miR-15a mimics, mimics NC, miR-15a inhibitor, and inhibitor NC was transfected into Yanbian yellow cattle precursor adipocytes, and the total cellular RNA was extracted. Protein detection of gene expression in pathways related to lipid metabolism was conducted. The results showed that overexpression of miR-15a reduced the expression of SREBP1, ACC, FAS, ADPN, AMPKa, and mTOR genes to varying degrees, while inhibiting the expression of miR-15a increased the expression of these genes in cells. With the amount of gene expression combined with the previous analysis of the results of PPARY and C/EBPa, we can conclude that miR-15a may participate in the AMPK-mTOR-Wnt pathway regulation of the differentiation and lipid production of pre-adipocytes by inhibiting the expression of the ABAT gene.

\section{Conclusions}

miR-15a can regulate the differentiation of Yanbian yellow cattle precursor adipocytes and overexpression of miR-15a reduces the lipid droplet content and lipid droplet size of Yanbian yellow cattle fat cells. Correspondingly, it also reduces the mRNA and protein expression of the lipid metabolism marker genes PPARY and C/EBPa. Meanwhile, inhibiting the expression of miR-15a has the opposite effect.

miR-15a targets the ABAT gene to regulate the adipocyte differentiation of Yanbian yellow cattle. Overexpressed miR-15a significantly inhibited the activity of the ABAT-3'UTR dual-luciferase reporter gene vector, indicating that miR-15a can have a targeting effect with ABAT gene. At the same time, the overexpression of miR-15a significantly reduced the expression of the ABAT gene in the cell. In contrast, inhibiting the expression of miR-15a will significantly increase the expression of the ABAT gene. 
miR-15a may regulate Yanbian yellow cattle adipocyte differentiation through the AMPK-mTOR-Wnt pathway. Overexpression of miR-15a reduces the expression of SREBP1, ACC, FAS, ADPN, AMPKa, and mTOR genes to varying degrees, while inhibiting the expression of miR-15a increases the expression of these genes in cells. With the amount of gene expression combined with the previous analysis of the results of PPARY and $\mathrm{C} / \mathrm{EBPa}$, we can conclude that miR-15a may participate in the regulation of the differentiation and lipid production of pre-adipocytes by inhibiting the expression of the ABAT gene in the AMPK-mTOR-Wnt pathway.

\section{Abbreviations}

miRNA: microRNA

ABAT: 4-aminobutyrate aminotransferase

PPARY: peroxisome proliferator-activated receptor $y$

C/EBPa: CCAAT enhancer-binding protein a (C/EBPa)

\section{Declarations}

\section{Acknowledgements}

We would like to thank Editage (www.editage.cn) for English language editing.

\section{Funding}

This study was supported by the Science and Technology Development Plan of Jilin Province of China (20200402053NC); National Natural Science Foundation of China (32160774); 111 Projrct (D20034).

\section{Author information}

\section{Affiliations}

Agriculture College, Yanbian University, Yanji, Jilin, 133002, China

Jinhui Bai, Hongyan Xu, Congcong Zhang, Jixuan Song, Xinxin Zhang, Beibei Hao, Baozhen Yin \& Guangjun Xia

Engineering Research Center of North-East Cold Region Beef Cattle Science \& Technology Innovation, Ministry of EducationपYanbian University, Yanji, 133002, China

Guangjun Xia

Faculty of Agriculture and Life Science, Hirosaki University, Hirosaki, Japan 
Jiachen Fang

\section{Contributions}

Hongyan Xu and Jinhui Bai equally contributed this work. HY X and JH B led the design and performance of the experiments, the analysis of the data,the writing of the paper, and the measurement of indicators. JC F, CC Z, JX S, XX Z, BB H, BZ Y \& GJ X perform the experiments and editing the paper. All authors have read and approved the final manuscript.

\section{Corresponding author}

Correspondence to Guangjun Xia.

\section{Ethics declarations}

\section{Ethics approval and consent to participate}

All of the experimental procedures for this experiment were performed according to the guideline established by the Regulation for the Administration of fairs Concerning Experimental Animals (Ministry of Science and Technology, China, 2004) and it was approved by the medical ethics committee of Yanbian university medical college (Approval ID: 201702).

\section{Consent for publication}

Not applicable.

\section{Competing interests}

No potential conflicts of interest relevant to this article were reported.

\section{References}

[1] Siersbæk R, Nielsen R, Mandrup S. Transcriptional networks and chromatin remodeling controlling adipogenesis. Trends Endocrinol Metab. 2012;23(2):56-64. doi:10.1016/j.tem.2011.10.001

[2] Bartel DP. MicroRNAs: genomics, biogenesis, mechanism, and function. Cell. 2004;116(2):281-297. doi:10.1016/s0092-8674(04)00045-5

[3] Bartel DP. MicroRNAs: target recognition and regulatory functions. Cell. 2009;136(2):215-233. doi:10.1016/j.cell.2009.01.002

[4] Williams MD, Mitchell GM. MicroRNAs in insulin resistance and obesity. Exp Diabetes Res. 2012;2012:484696. doi:10.1155/2012/484696 
[5] Ji H, Chai Z, Wang H, Zhong J.Research Progress on miRNA in Bovine Adipose Tissue. China Herbivore Science. 2018,38(04):53-57.

[6] Fan Y, Arbab AAl, Zhang H, et al. MicroRNA-193a-5p Regulates the Synthesis of Polyunsaturated Fatty Acids by Targeting Fatty Acid Desaturase 1 (FADS1) in Bovine Mammary Epithelial Cells. Biomolecules. 2021;11(2):157. Published 2021 Jan 25. doi:10.3390/biom11020157

[7] Jin W, Dodson MV, Moore SS, Basarab JA, Guan LL. Characterization of microRNA expression in bovine adipose tissues: a potential regulatory mechanism of subcutaneous adipose tissue development. BMC Mol Biol. 2010;11:29. Published 2010 Apr 27. doi:10.1186/1471-2199-11-29

[8] Wang M, Zhou C, Cong S, et al. Lipopolysaccharide inhibits triglyceride synthesis in dairy cow mammary epithelial cells by upregulating miR-27a-3p, which targets the PPARG gene. J Dairy Sci. 2021;104(1):989-1001. doi:10.3168/jds.2020-18270

[9] Chen X, Raza SHA, Cheng G, Ma X, Wang J, Zan L. Bta-miR-376a Targeting KLF15 Interferes with Adipogenesis Signaling Pathway to Promote Differentiation of Qinchuan Beef Cattle Preadipocytes. Animals (Basel). 2020;10(12):2362. Published 2020 Dec 10. doi:10.3390/ani10122362

[10] Kamalden TA, Macgregor-Das AM, Kannan SM, et al. Exosomal MicroRNA-15a Transfer from the Pancreas Augments Diabetic Complications by Inducing Oxidative Stress. Antioxid Redox Signal. 2017;27(13):913-930. doi:10.1089/ars.2016.6844

[11] Hu X: Investigation of $\mathrm{H}_{2} \mathrm{~S}$ Content in Chicken Farms in a Certain and Effect of $\mathrm{H}_{2} \mathrm{~S}$ on Chicken Production Performance and Immune Function. https://kns.cnki.net/KCMS/detail/detail.aspx? dbname=CDFDLAST2021\&filename=1020078372.nh (2020). Accessed 1 Jun 2020.

[12] Guo Y, Li G, Li H, et al. MicroRNA-15a Inhibits Glucose Transporter 4 Translocation and Impairs Glucose Metabolism in L6 Skeletal Muscle Via Targeting of Vesicle-Associated Membrane ProteinAssociated Protein A. Can J Diabetes. 2020;44(3):261-266.e2. doi:10.1016/j.jcjd.2019.07.151

[13] Zhao G, Wang X. The research progress of ABAT gene in human diseases. Fudan University Journal of Medical Sciences. 2016;43(03):357-361+367.

[14] Chen Z: Expression of ABAT in clear cell renal cell carcinoma and its effect on the proliferation, migration and metabolism of renal carcinoma cell lines. https://kns.cnki.net/KCMS/detail/detail.aspx? dbname=CMFD201902\&filename=1018166926.nh (2018). Accessed 1 May 2018.

[15] Sun L, Lu S, Bai M, et al. Integrative microRNA-mRNA Analysis of Muscle Tissues in Qianhua Mutton Merino and Small Tail Han Sheep Reveals Key Roles for oar-miR-655-3p and oar-miR-381-5p. DNA Cell Biol. 2019;38(5):423-435. doi:10.1089/dna.2018.4408 
[16] Xia G: Screening of candidate genes associated to meat quality traits of Yanbian Yellow Cattle by a Combination of miRNA and Functional Genes Transcriptome.

https://kns.cnki.net/KCMS/detail/detail.aspx?dbname=CDFDLAST2015\&filename=1014359717.nh (2014). Accessed 10 Apr 2014.

[17] Shao J: Effects of miR-1271, miR-381, miR-17-3P and miR-22-3P on Differentiation of Yanbian Yellow Cattle Precursor Adipocytes. https://kns.cnki.net/KCMS/detail/detail.aspx?

dbname=CMFD202101\&filename=1020075290.nh (2020). Accessed 28 May 2020.

[18] Fernyhough ME, Vierck JL, Hausman GJ, Mir PS, Okine EK, Dodson MV. Primary adipocyte culture: adipocyte purification methods may lead to a new understanding of adipose tissue growth and development. Cytotechnology. 2004;46(2-3):163-172. doi:10.1007/s10616-005-2602-0

[19] Xu H, Shao J, Yin B, et al. Bovine bta-microRNA-1271 Promotes Preadipocyte Differentiation by Targeting Activation Transcription Factor 3. Biochemistry (Mosc). 2020;85(7):749-757. doi:10.1134/S0006297920070032

[20] Xu H, Shao J, Fang J, et al. miR-381 Targets KCTD15 to Regulate Bovine Preadipocyte Differentiation In Vitro. Horm Metab Res. 2021;53(1):63-70. doi:10.1055/a-1276-1602

[21] Wang X, Li Y, Qu L, et al. Lipolytic gene DAGLA is targeted by miR-223 in chicken hepatocytes. Gene. 2021;767:145184. doi:10.1016/j.gene.2020.145184

[22] Lee S, Park SJ, Cheong JK, Ko JY, Bong J, Baik M. Identification of circulating miRNA involved in meat yield of Korean cattle. Cell Biol Int. 2017;41(7):761-768. doi:10.1002/cbin.10776

[23] Li Z, Zhang H, Song N, et al. Molecular cloning, characterization and expression of miR-15a-3p and miR-15b-3p in dairy cattle. Mol Cell Probes. 2014;28(5-6):255-258. doi:10.1016/j.mcp.2014.06.003

[24] Li H, Wang C, Li Q, Gao X. MiR-15a decreases bovine mammary epithelial cell viability and lactation and regulates growth hormone receptor expression. Molecules. 2012;17(10):12037-12048. Published 2012 Oct 12. doi:10.3390/molecules 171012037

[25] Wu X: Roles of Wnt/ß-catenin Signaling in Adipogenic Differentiation of Poultry Mesenchymal Stem Cells. https://kns.cnki.net/KCMS/detail/detail.aspx?dbname=CMFD201902\&filename=1019103855.nh (2019). Accessed 1 May 2019.

[26] Yi J, Zhu J, Wu J, Thompson CB, Jiang X. Oncogenic activation of PI3K-AKT-mTOR signaling suppresses ferroptosis via SREBP-mediated lipogenesis. Proc Natl Acad Sci U S A. 2020;117(49):3118931197. doi:10.1073/pnas.2017152117

[27] Guo Y, et al. Lipid deposition in abalone Haliotis discus hannai affected by dietary lipid levels through AMPKa2/PPARa and JNK/mTOR/SREBP-1c pathway. Aquaculture. 2021;532:736040. doi: 10.1016/j.aquaculture.2020.736040. 
[28] Luo Z, Song Q, Xiong X, et al. The PI3K/Akt/mTOR signaling pathway regulates lipid metabolism mediated by endoplasmic reticulum stress in goose primary hepatocytes. ResearchGate. 2021.

doi: 10.1399/eps.2021.325

[29] Zhang AR, Sun J, He Y, Wang N, Tian L. Attenuation of lipid accumulation in Bel-7402 cells through ADPN/AMPKa signaling stimulated by Fructus rosae laxae extract. J Food Biochem.

2020;44(12):e13497. doi:10.1111/jfbc.13497

[30] Tan L, Song A, Ren L, Wang C, Song G. Effect of pioglitazone on skeletal muscle lipid deposition in the insulin resistance rat model induced by high fructose diet under AMPK signaling pathway. Saudi J Biol Sci. 2020;27(5):1317-1323. doi:10.1016/j.sjbs.2020.03.014

[31] Yao J, Wang Z, Cheng Y, et al. M2 macrophage-derived exosomal microRNAs inhibit cell migration and invasion in gliomas through PI3K/AKT/mTOR signaling pathway [published correction appears in $\mathrm{J}$ Transl Med. 2021 Jun 16;19(1):263]. J Transl Med. 2021;19(1):99. Published 2021 Mar 6. doi:10.1186/s12967-021-02766-w

[32] Zhang W, Li Y, Xi X, et al. MicroRNA-15a-5p induces pulmonary artery smooth muscle cell apoptosis in a pulmonary arterial hypertension model via the VEGF/p38/MMP-2 signaling pathway. Int J Mol Med. 2020;45(2):461-474. doi:10.3892/ijmm.2019.4434

\section{Figures}

$\begin{array}{ll}\text { bta-miR-15a } & \text { UAGCAGCACAUAAUGGUUUGU } \\ \text { hsa-miR-15a-5p } & \text { UAGCAGCACAUAAUGGUUUGUG } \\ \text { ppy-miR-15a } & \text { UAGCAGCACAUAAUGGUUUGUG } \\ \text { ptr-miR-15a } & \text { UAGCAGCACAUAAUGGUUUGUG } \\ \text { ssc-miR-15a } & \text { UAGCAGCACAUAAUGGUUUGU } \\ \text { mm } 1-m i R-15 a-5 p & \text { UAGCAGCACAUAAUGGUUUGUG } \\ \text { eca-miR-15a } & \text { UAGCAGCACAUAAUGGUUUGUG } \\ \text { chi-miR-15a-5p } & \text { UAGCAGCACAUAAUGGUUUGUGG } \\ \text { ggo-miR-15a } & \text { UAGCAGCACAUAAUGGUUUGUG } \\ \text { cfa-miR-15a } & \text { UAGCAGCACAUAAUGGUUUGU }\end{array}$

Figure 1

Comparison of miR-15a sequences among different species (yellow region is seed sequence) bta: Bos Taurus; hsa: Homo sapiens; ppy: Pongo pygmaeus; ptr: Pan troglodytes; ssc: Sus scrofa; mml: Macaca mulatta; eca: Equus caballus; chi: Capra hircus; ggo: Gorilla; cfa: Canis familiaris. 
a

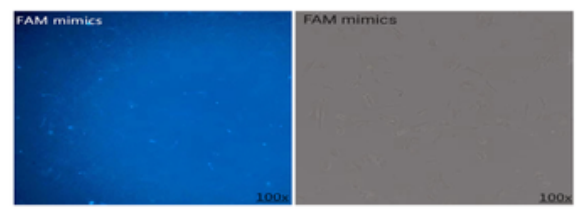

b
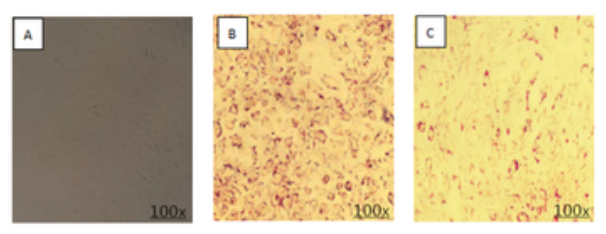

c

d
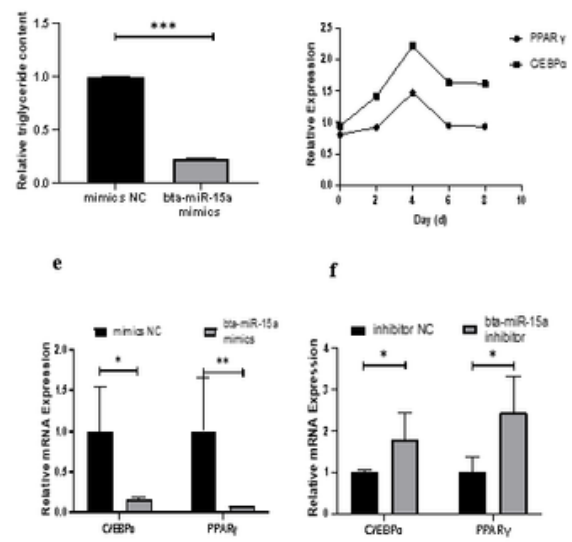

f

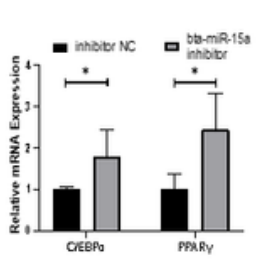

g

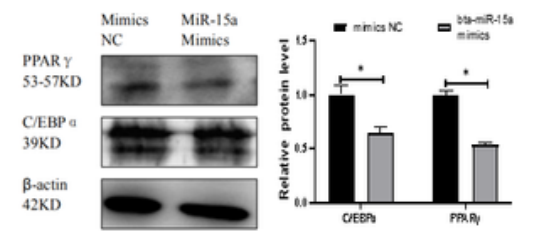

h

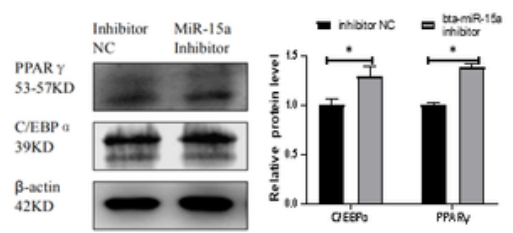

\section{Figure 2}

miR-15a can regulate the differentiation of Yanbian yellow cattle precursor adipocytes. a Detection of miR-15a transfection efficiency. $b$ Effect of miR-15a on lipid formation during the differentiation of Yanbian yellow cattle precursor adipocytes. (A) Before induced differentiation (B) Oil red 0 staining in mimics NC control group (C) bta-miR-15a mimics oil red O staining. c Effect of miR-15a on TG content in Yanbian yellow cattle precursor adipocytes. d Expression of adipogenic marker genes PPARy and C/EBPa 
during the differentiation of Yanbian yellow cattle precursor adipocytes. e Effect of overexpression of miR-15a on mRNA expression of lipid metabolism marker genes in Yanbian yellow cattle precursor adipocytes. $f$ Effects of inhibiting miR-15a expression on PPARY and C/EBPa mRNA expression in Yanbian yellow cattle precursor adipocytes. $g$ Effects of overexpression of miR-15a on PPARY and $\mathrm{C} / \mathrm{EBPa}$ protein expression in Yanbian yellow cattle precursor adipocytes. $\mathrm{h}$ Effects of inhibition of miR15a expression on PPARy and C/EBPa protein expression in bovine precursor adipocytes.

a

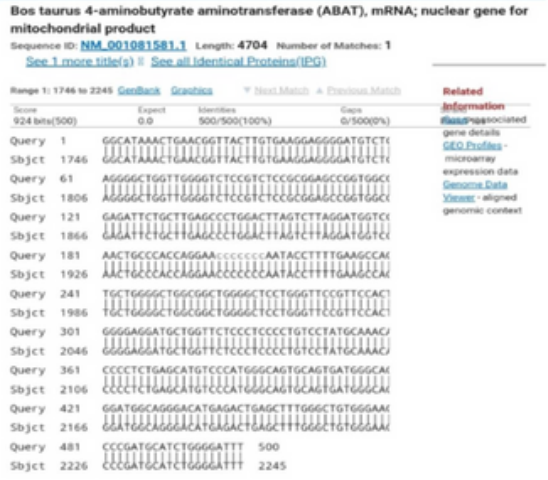

b
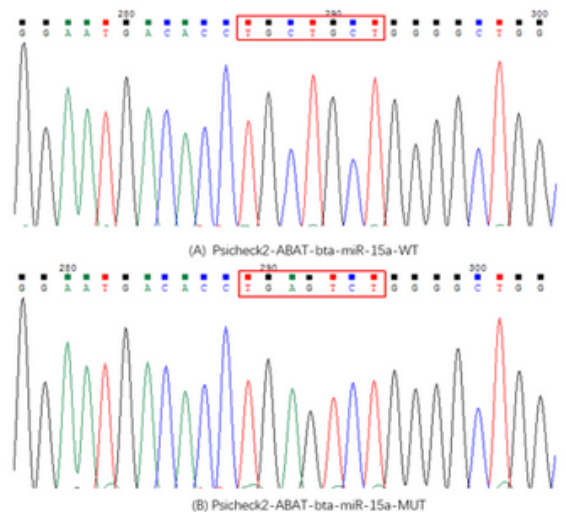

- mimics NC 口 miR-15a mimics

c

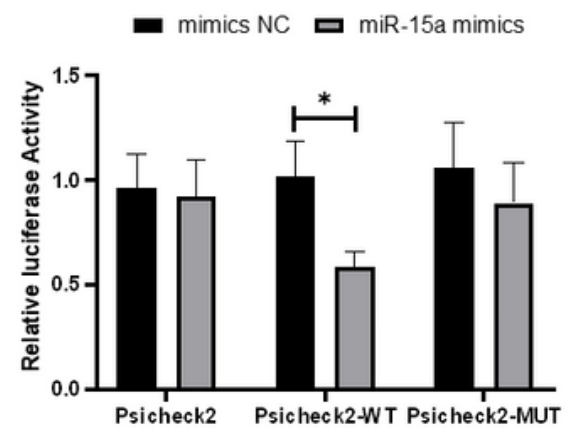

Figure 3 
miR-15a targets the 3'UTR of the ABAT gene of cattle. a The alignment of the insert sequence with the sequence of ABAT. b Carrier sequencing. (A) Wild type carrier; (B) Mutant carrier. $c$ Detection results of miR-15a and ABAT targeting relationship.

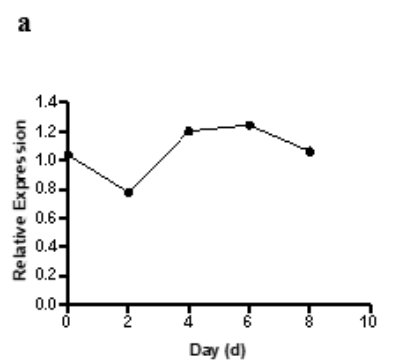

b

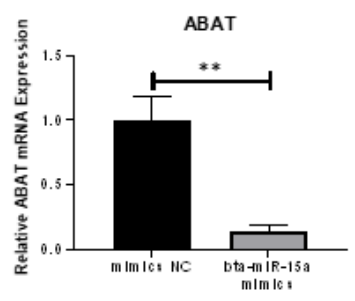

c
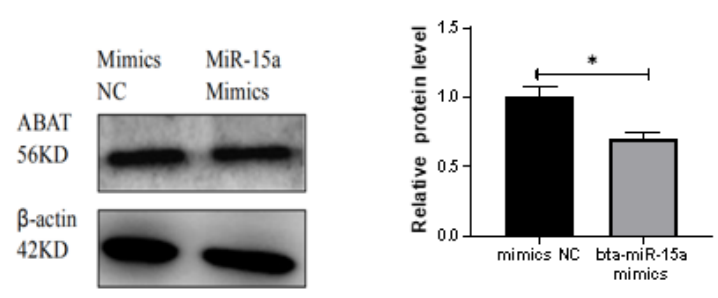

d

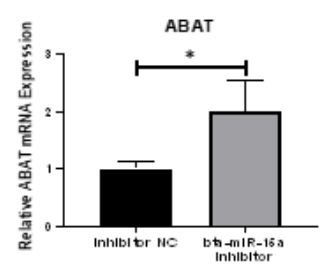

e

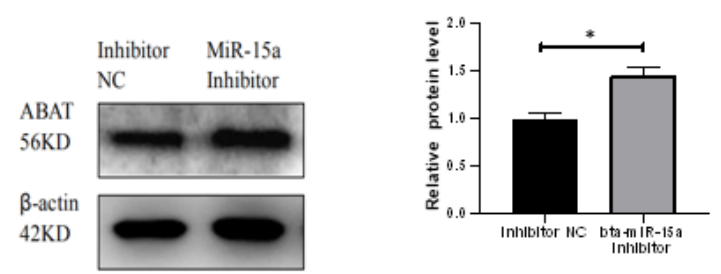

Figure 4

miR-15a targets ABAT gene to regulate the differentiation of Yanbian yellow cattle adipocytes. a The expression level of ABAT gene changed with different induction time of cell differentiation. b Effect of 
overexpression of miR-15a on mRNA expression of ABAT gene. c Effect of overexpression of miR-15a on protein expression of ABAT gene. d Effect of inhibition of miR-15a expression on ABAT mRNA expression. e Effect of inhibition of miR-15a expression on protein expression of ABAT gene.
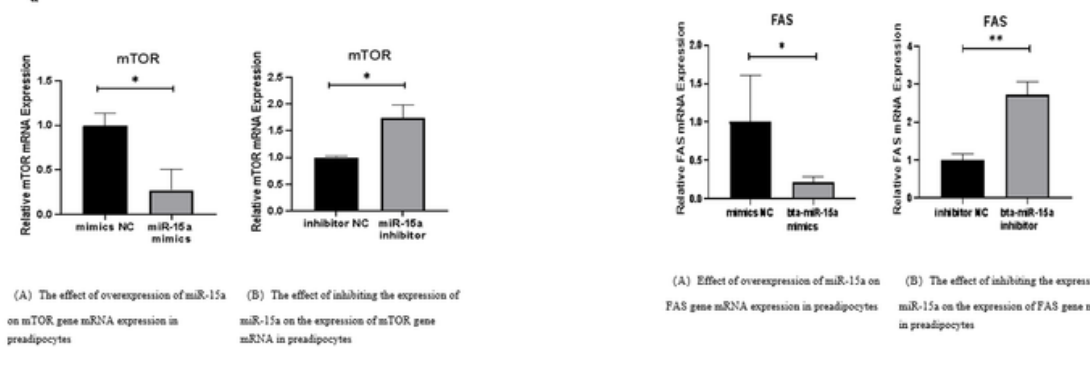

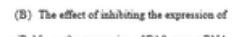

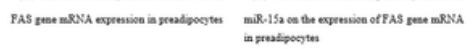

b
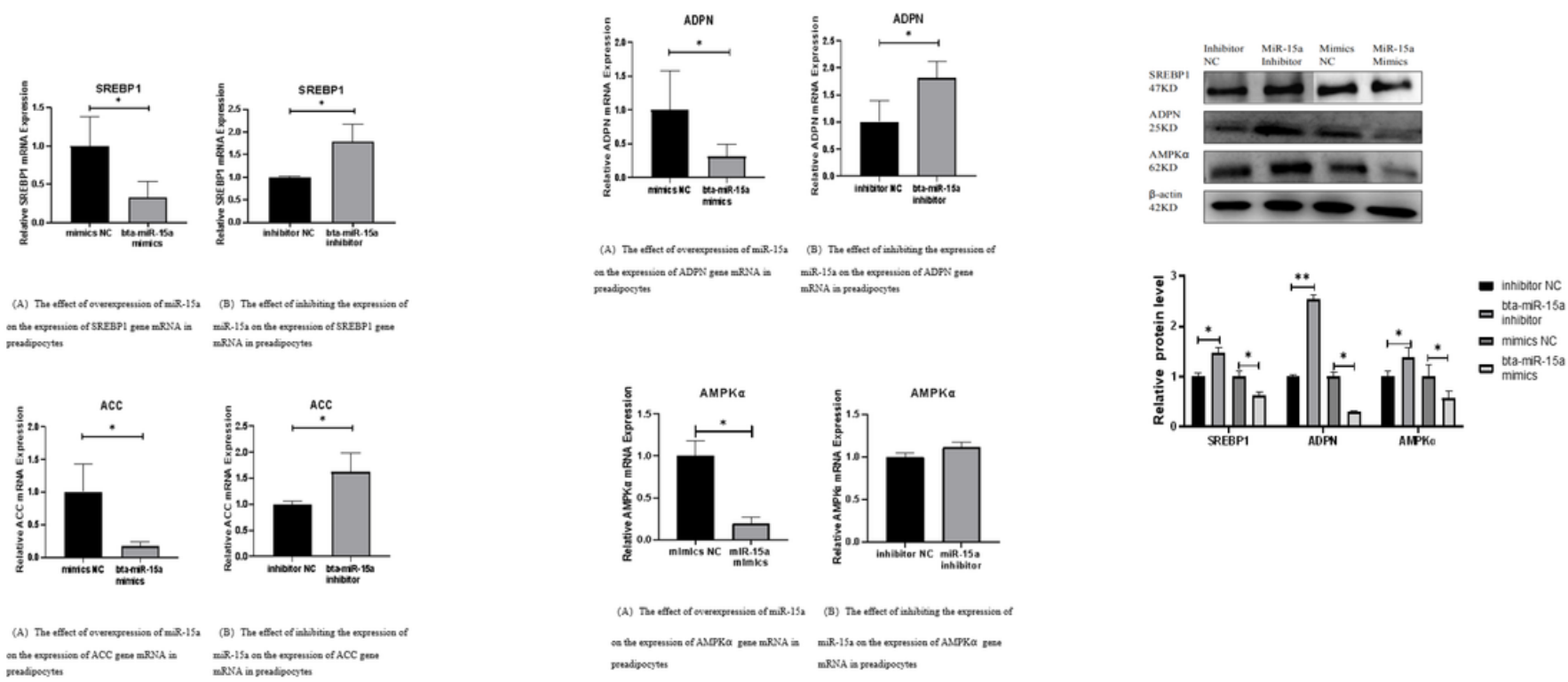

\section{Figure 5}

miR-15a participates in AMPK-mTOR-Wnt pathway to regulate the differentiation of Yanbian yellow cattle precursor adipocytes. a Effects of miR-15a on genes related to lipid metabolic pathway in bovine preadipocytes. b Effects of miR-15a on the expression of gene proteins related to lipid metabolism pathway in bovine precursor adipocytes.

\section{Supplementary Files}

This is a list of supplementary files associated with this preprint. Click to download.

- Supplementarylnformation.docx 